\title{
ENFERMEDAD DE COATS RECIDIVANTE DIAGNOSTICADA EN SUJETO ADULTO
}

\section{ADULT-ONSET OF RECURRENT COATS DISEASE}

\author{
VÁZQUEZ PULIDO N${ }^{1}$, LALIENA SANTAMARÍA JL ${ }^{2}$, DEL BUEY SAYAS MA ${ }^{1}$, \\ BURDEUS GOMEZ R ${ }^{1}$, OSAN TELLO M${ }^{1}$
}

\section{RESUMEN}

Caso clínico: Paciente varón que con 30 años tuvo el primer episodio de enfermedad de Coats y recurrencia 14 años más tarde. Clínicamente ambos episodios tienen como síntoma de inicio visión borrosa y exploración típica de exudación masiva retiniana con corona de exudados duros. La angiografía presenta hallazgos característicos de telangiectasia retiniana con dilatación anormal de vasos con hiperfluorescencia precoz y fuga tardía.

Discusión: Coats es una entidad clínica poco frecuente y con grado de severidad variable, que hacen difíciles los estudios randomizados prospectivos. Pese a los hallazgos característicos, hay que descartar otros diagnósticos tanto en el niño como en el adulto causantes de esa misma clínica.

Palabras clave: Enfermedad de Coats, teleangectasias retinianas, exudación retiniana, recidiva tarda, disminución de agudeza visual.

\begin{abstract}
Case report: A male diagnosed with Coats disease at the age of 30 years, had a relapse of this condition 14 years later. His first symptom of blurred vision occurred in both episodes and similar findings of unilateral retinal exudation and lipid deposition at boundary were seen. Angiography showed the characteristic early hyperfluorescence of the telangiectasias and late leakage of dye.
\end{abstract}

Discussion: Coats disease is relatively uncommon and, as the degree of involvement varies from case to case, a randomized, prospective study of treatment would probably not be feasible. Despite the characteristic features of this disease, the differential diagnoses must be considered in both children and adults (Arch Soc Esp Oftalmol 2007; 82: 555 558).

Key words: Coats disease, retinal telangiectasias, retinal exudation, late recurrence, decreased visual acuity.

\footnotetext{
Recibido: 8/9/06. Aceptado: 17/7/07.

Hospital General San Jorge. Huesca. España.

1 Licenciado en Medicina.

2 Doctor en Medicina.

Correspondencia:

Nuria Vázquez Pulido

Marceliano Isabal, 3, 5 . $^{\circ}$

50004 Zaragoza

España

E-mail: nuria_vazquez@hotmail.com
} 


\section{INTRODUCCIÓN}

Se presenta un caso atípico de recidiva de enfermedad de Coats en la edad adulta. Descrita por George Coats en 1908, es una vasculopatía idiopática unilateral, caracterizada por la presencia de telangectasias, exudación masiva y desprendimiento de retina (DR) exudativo. Es más frecuente en varones y típicamente unilateral. No se ha demostrado carácter hereditario o asociación con enfermedades sistémicas. Suele diagnosticarse en la primera década de la vida $(1,2)$. Generalmente, es más agresiva cuanto menor es la edad inicial de presentación (3).

\section{CASO CLÍNICO}

Varón de 44 años que acude a Urgencias por presentar visión borrosa en su ojo derecho (OD). El paciente había sido revisado en nuestro servicio en 1990, hacía 14 años, por presentar un primer episodio visión borrosa. La agudeza visual (AV) fue 9/10 en OD y unidad OI. Exploración sin hallazgos, excepto en fondo de ojo (FO) que mostró exudación, microhemorragias, así como discreto DR exudativo en región peripapilar y temporal inferior. La enfermedad de Coats fue diagnóstico de sospecha, pese a la presentación atípica en sujeto adulto, por el aspecto funduscópico, la angiografía (AGF) y ausencia de antecedentes personales que se relacionaran con retinopatía exudativa unilateral. El estudio general (analítica sanguínea, serología y radiografía de tórax) no mostró alteraciones. Se realizó fotocoagulación láser argón, que se aplicó en 3 sesiones. Los exudados se reabsorbieron completamente en un año y medio, manteniéndose AV de la unidad y quedando solo la cicatriz de laser (fig. 1).

En su nuevo episodio de visión borrosa 14 años después, la AV fue 9/10 en su OD. Polo anterior normal y PIO de $16 \mathrm{mmHg}$ en AO. El FO OD reveló DR exudativo nasal amplio rodeado de exudados duros, microhemorragias y estrella macular (fig. 2). La AGF OD mostró la característica hiperfluorescencia precoz de telangectasias y microaneurismas (fig. 3), con fuga tardía (fig. 4). Se trató con fotocoagulación láser, en tres sesiones espaciadas a lo largo de dos años, mejorando paulatinamente (fig. 5) hasta la resolución completa (fig. 6). La exploración de OI fue normal en ambos episodios. Actualmente asintomático con AV de unidad.

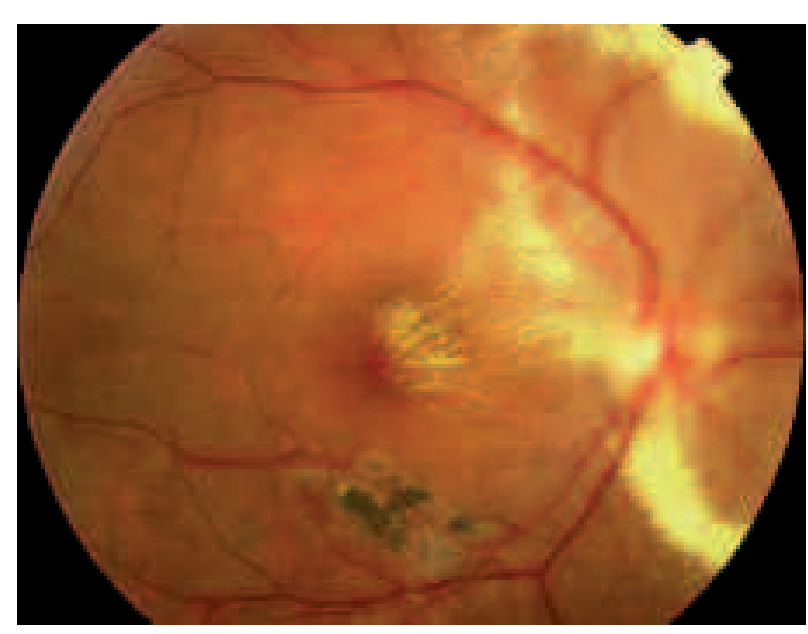

Fig. 1: En la oftalmoscopía del OD destacan exudación peripapilar y cicatriz de antiguo episodio fotocoagulado.

\section{DISCUSIÓN}

La presentación típica de Coats, se observa en niños que presentan disminución de AV, estrabismo o leucocoria. Shields, en una serie de 150 casos, observó la mediana de la edad de diagnosticó de 5 años; aunque con rango de 1 mes a 63 años, es infrecuente diagnosticarla inicialmente en adultos. (2). En ambos casos, se afecta principalmente la vascularización. En adultos, las alteraciones aparecen en la región ecuatorial y periférica, son más frecuentes las hemorragias asociadas a macroaneurismas, y la evolución es de progresión más lenta que en niños (3). En la enfermedad de Coats podría

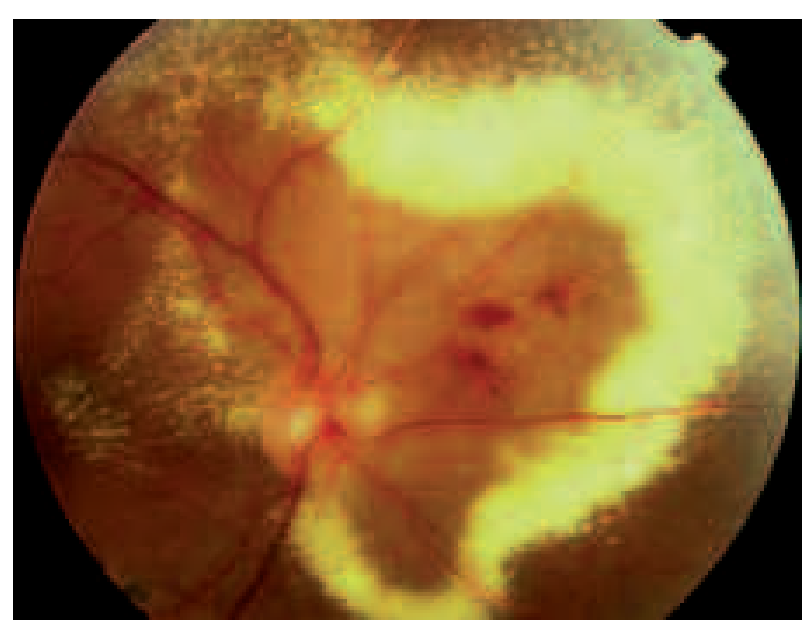

Fig. 2: Exudación masiva en sector nasal, DR exudativo y estrella macular. 


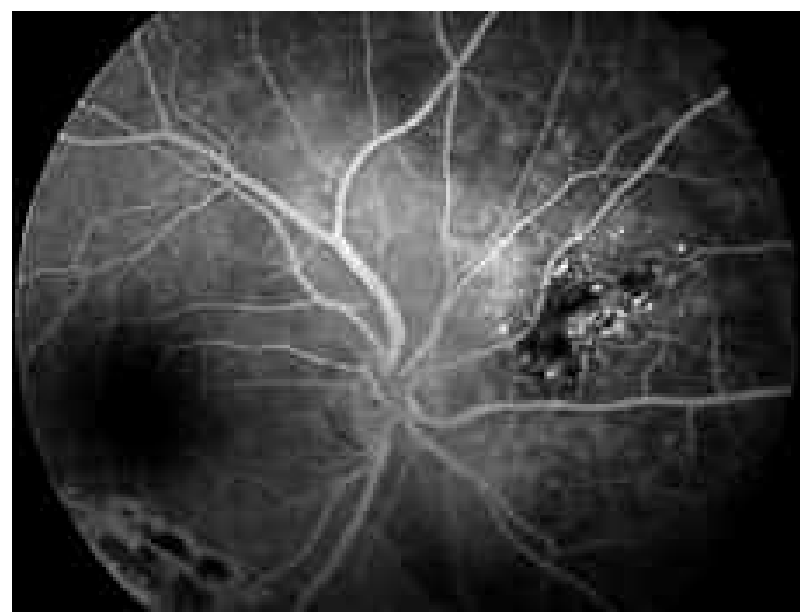

Fig. 3: AGF OD: hiperfluorescencia de los vasos anormales retinianos.

estar afectada la proliferación y migración de las células endoteliales debido a mutaciones de genes responsables de la proteína norrina. Es probable que diferentes defectos den lugar a diferentes fenotipos de la enfermedad, con diferentes grados de severidad y edad de comienzo $(3,4)$.

En nuestro caso no existía historia previa de DR, diabetes, exposición a radiación, episodios de inflamación intraocular, degeneración tapetorretiniana, oclusión vascular. Todas estas enfermedades pueden tener una respuesta retiniana exudativa, similar al aspecto clínico de la enfermedad de Coats, conocido como Coats-like syndrome (3). Gass, clasifica en un grupo diferente a los sujetos con hipertensión arterial, policitemia vera, corona-

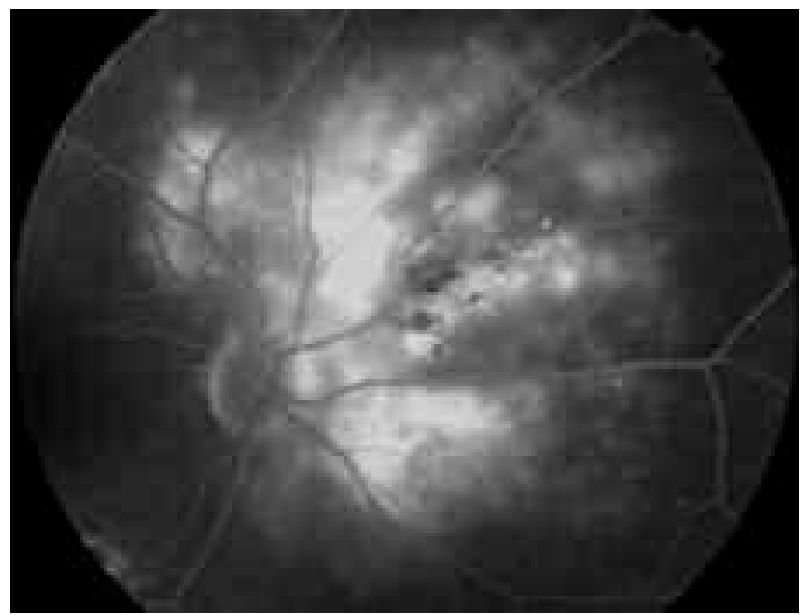

Fig. 4: AGF OD: fase tardía mostrando fuga y tinción intraretinal y subretinal de fluido.

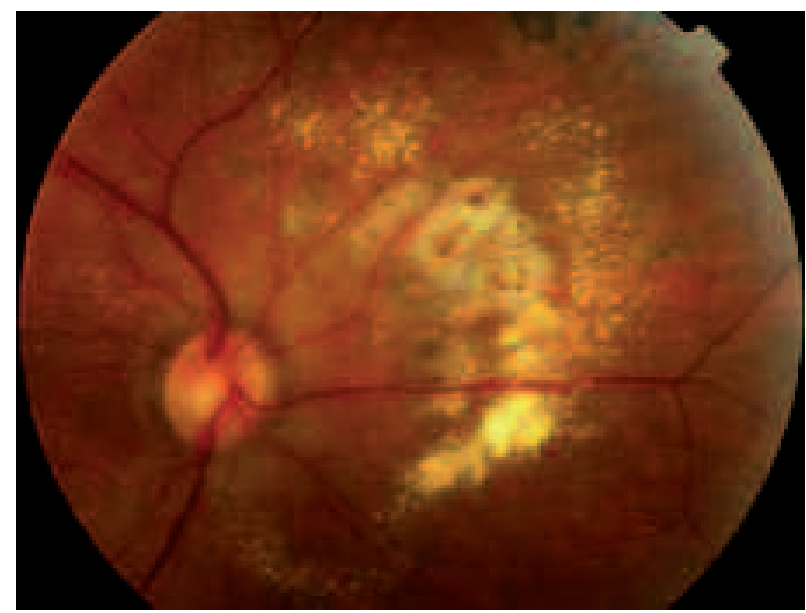

Fig. 5: FO OD: reabsorción paulatina de los exudados, tras fotocoagulación láser.

riopatía y diabetes, aunque considere idiopáticas las telangectasias (4).

Shields clasificó la enfermedad atendiendo al tipo y localización de las lesiones (2). Si sólo se observan telangectasias retinianas se trata de un estadio 1. Si además hay exudación es estadio 2 (2A, extrafoveal o 2B, foveal). El estadio 3 lo define la presencia de DR exudativo y el estadio 4 asocia DR total y glaucoma secundario.

Es complicado realizar estudios prospectivos y randomizados sobre esta enfermedad y las diferentes opciones de tratamiento, ya que es poco frecuente y su severidad muy variable. El objetivo del tratamiento es obliterar las telangectasias para detener la exudación y reaplique del DR si éste se pro-

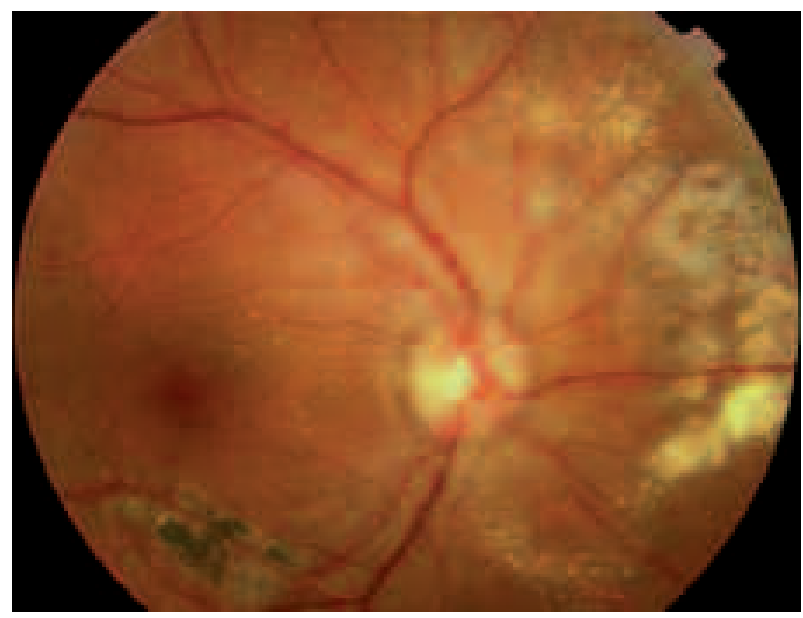

Fig. 6: FO OD: retina reaplicada, sin exudación. Se aprecian marcas de láser y atrofia retina sector nasal. 
dujo. En estadios precoces puede lograrse mediante fotocoagulación con láser o crioterapia. Los casos más avanzados exigen además cirugía del DR. La fotocoagulación láser debe considerarse inicialmente con telangiectasias que se encuentran en periferia y con áreas de exudación extensa (1). Sin tratamiento la progresión natural de la enfermedad puede conducir a complicaciones como DR total bulloso, glaucoma neovascular y ptisis bulbi (5). Si el área de exudación se limita a un solo cuadrante o se localiza en sector nasal, el pronóstico visual es más favorable.

Se recomienda esperar al menos 3 meses para evaluar la eficacia del tratamiento láser porque la exudación se reabsorbe muy lentamente, como en nuestro paciente. Según hallazgos clínicos, se puede repetir tratamiento (1). La exudación y/o líquido subretiniano pueden conducir a un proceso de fibrosis irreversible que condiciona el pronóstico visual en casos de afectación macular. Se han descrito recidivas de las telangectasias y exudación en el 7\% de los casos tras 10 años después de un tratamiento satisfactorio $(1,5)$, que pueden ser múltiples, en intervalos variables de tiempo, con lo que el segui- miento debe ser de por vida. (en niños cada 6 meses aproximadamente (5), por la falta de queja y posibilidad de desarrollar ambliopía). A nivel de clínica no existe ni leucocoria ni estrabismo en el caso de adultos y suele ser bastante asintomático, y tampoco tiene complicaciones mayores como glaucoma neovascular (3).

\section{BIBLIOGRAFÍA}

1. Shields JA, Shields CL, Honavar SG, Demirci H. Clinical variations and complications of Coats disease in 150 cases: the 2000 Sanford Gifford Memorial Lecture. Am J Ophthalmol 2001; 131: 561-571.

2. Shields JA, Shields CL, Honavar SG, Demirci H, Cater J. Classification and management of Coats disease: the 2000 Proctor Lecture. Am J Ophthalmol 2001; 131: 572-583.

3. Smithen LM, Brown GC, Brucker AJ, Yannuzzi LA, Klais CM, Spaide RF. Coats' disease diagnosed in adulthood. Ophthalmology 2005; 112: 1072-1078.

4. Cahill M, O'Keefe M, Acheson R, Mulvihill A, Wallace D, Mooney D. Classification of the spectrum of Coats' disease as subtypes of idiopathic retinal teangiectasis with exudation. Acta Ophthalmol Scand 2001; 79: 596-602.

5. Shienbaum G, Tasman WS. Coats disease: a lifetime disease. Retina 2006; 26: 422-424. 